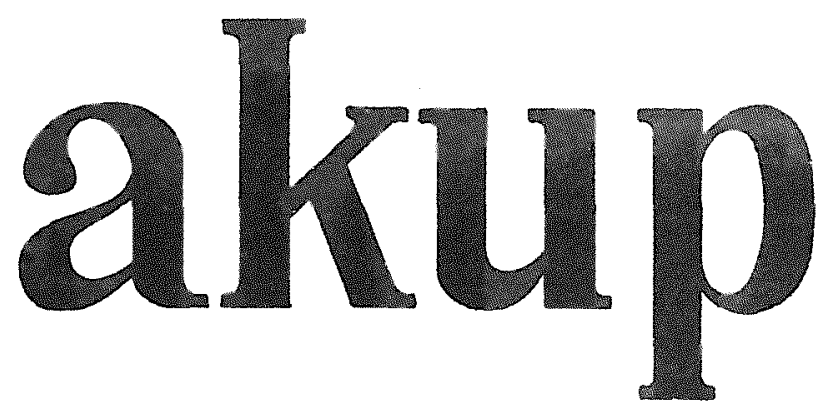

Arbeiten des Kölner Universalien - Projekts

Nr. 54

AKKUSATIVISCHE, ERGATIVISCHE UND AKTIVISCHE BEZEICHNUNG VON AKTANTENFUNKTIONEN

Klaus Heger

November 1983 
Herausgeber der Reihe:

Prof. Dr. Hansjakob Seiler

Universalienprojekt

Institut für sprachwissenschaft

Universität

D - 5000 Köln 41

(C) bei den Autoren 


\title{
AKKUSATIVISCHE, ERGATIVISCHE UND AKTIVISCHE BEZEICHNUNG VON AKTANTENFUNKTIONEIJ
}

\author{
Kleus Heger
}

O. Das Ziel der folgenden Betrachtungen besteht weniger in der Lieferung eines prinzipiell neuen Beitrags sei es zur Unterscheidung akkusativischer, ergativischer und aktivischer Konstruktionstypen oder sei es zu derjenigen verschiedener Aktantenfunktionen (cf. Heger 1976: $\$$ 4.1.2.) als vielmehr im Nachweis der Brauchbarkeit der letzteren als eines noematischen - d.h. von je einzelsprachlichen Gegebenheiten unabhängigen - tertium comparationis für den Vergleich der ersteren und ihrer verschiedenen Sub-Typen. Im Rahmen dieser Zielsetzung ist es legitim, die folgenden Be-trachtungen auf die Frage nach den Bezeichnungen der Prädikativ-Funktion (die einem Aktanten eine Eigenschaft, einen Zustand oder eine Zustandsveränderung zuschreibt), der Kausal-Funktion (die einen Aktanten als denjenigen theoreti-.. schen Ort charakterisiert, von dem die Ursache eines Zustands oder Vorgangs ausgeht) und der Final-..Funktion (die einen Aktanten als denjenigen theoretischen ort charakterisiert, an dem sich die Wirkung eines Zustancis oder Vorgangs vollzieht) einzuschränken. Dennoch ist der einleitende Hinweis auf die zumindest theoretisch unbegrenzte Unterscheidbarkeit weiterer Aktantenfunktionen wichtig, da er die naheliegende Frage beantwortet, warum in keiner sprache einein... deutige Bezeichnungen von Aktantenfunktionen anzutreffen sind: im Fall der Bezeichnung von Aktantenfunktionen durch Kasusgramrieme am IIomen würde eine derartige Lösung kaum mehr handhabbare Flexionsparadignen entstehen lassen und im Fall ihrer Bezeichnung durch Kongruenzmarkierung am Verb darúberhinaus zu monströsen Konjugationsformen führen. 
1. Angesichts dieses Zwangs zu ökonomischeren Lösungen kann es nicht überraschen, daß praktisch alle sprachen in irgendeiner Weise davon profitieren, daß das Fehlen einer eindeutigen Bezeichnung einer Aktantenfunktion nur dort zu Verwechslungsgefahren führt, wo die in paradigmatischer opposition stehenden Aktantenfunktionen auch in syntagmatischem Kortrast erscheinen. Die Frage nach je einzelsprachlichen Bezeichnungen für die Prädikativ-, die Kausal- und die FinalFunktion hat somit nicht nur diese drei Aktantenfunktionen selbst zu unterscheiden, sondern muß daruberhinaus auch berücksichtigen, ob und, wenn ja, in welchen syntagmatischen Kontrasten sie jeweils auftreten können. Bei der in einem ersten Schritt vorzuführenden ausschließlichen Berucksichtigung der Prädikativ- und der Kausal-Funktion sind daher statt zweier vier Fälle zu unterscheiden, je nach dem ob

- die Prädikativ-Funktion in syntagmatischer Isolierung (im folgenden markiert als: $\mathrm{P}$ ),

- die Prädikativ-Funktion in syntagmatischem Kontrast mit der Kausal-Funktion (im folgenden: $P(C)$ ),

- die Kausal-Funktion in syntagmatischer Isolierung (im folgenden: C ) und/oder

- die Kausal-Funktion in syntagmatischem Kontrast mit der Prädikativ-Funktion (im folgenden: C (P) )

auftreten. Entsprechend sind bei der in einem zweiten schritt vorzunehmenden Einbeziehung der Final-Funktion statt dreier zwölf verschiedene Fälle zu unterscheiden:

- für die Prädikativ-Funktion $P, P(C), P(F), P(C, F)$,

- für die Kausal-Funktion $C, C(P), C(F), C(P, F)$ und

- fir die Final-Funktion $F, F(P), F(C), F(P, C)$.

Ein besonderes Problem wirft hierbei die Unterstellung der Möglichkeiten C (Kausal-Funktion in syntagmatischer Isolierung), $C(F)$ (Kausal-Funktion in syntagmatischem Kontrast nur mit Final-Funktion), F (Final-Funktion in syntagmatischer Isolierung) und $F(C)$ (Final-Funktion in syntagmatischem Kon- 
trast nur mit Kausal-Funktion) auf. Es sind dies diejenigen Fälle, in denen syntagmatisch keine Prädikativ-Funktion erscheint und die somit der Definition der Kausal- und der Final-Funktion durch ihre Stellung im Rahmen von Aktantenmodellen, die außer einem Aktanten in Kausal-bzw. FinalFunktion mindestens einen weiteren in Prädikativ-Funktion enthalten, zu widersprechen scheinen. Mindestens die beiden folgenden Möglichkeiten zwingen dennoch zur Berücksichtigung dieser Fälle.

1.1. Es kann sich um Aktantenmodelle handeln, deren unterstem Prädikator (oder Temporal-Funktor) ein Aktant in Prädikativ-Funktion untergeordnet ist, der schon durch das - ohne zusätzliche Unterordnung unter weitere Aktantenmodell-Komponenten: avalente - Verballexem selbst bezeichnet wird und daher syntagmatisch nicht nochmals eigens zu erscheinen braucht; Beispiele hierfir sind Juppiter pluit (mit Juppiter in Kausal-Funktion) oder mir ist kalt (mit mir in Final-Funktion).

1.2. Es kann sich um Aktantenmodelle mit mehreren Aktanten-positionen und demzufolge mehreren Aktantenfunktionen handeln, bei denen eine referenzidentische Besetzung dieser Positionen schon implizit durch ein entsprechendes Verballexem bezeichnet wird, welches daher auch bei mehreren Aktantenfunktionen nur eine einzige Aktantenbezeichnung erfordert; Beispiele hierfü sind John is swimming (mit John in Prädikativ- und Kausal-Funktion, im Gegensatz zu fohn is floating mitelohn ausschlieBlich in Prädikativ-Funktion) oder Hans betrachtet den Baum (mit Hans in Kausal- und FinalFunktion, im Gegensatz zu Hans sieht den Baum mit Hans ausschließlich in Final-Funktion). Sowohl das, was sich theoretisch aus der Struktur von Aktantenmodellen ablesen läßt, als auch die praktische Erfahrung mit einer größeren AnzahI von Sprachen legen es nahe, in derartigen Fällen einer Kumulation mehrerer Aktantenfunktionen auf einen einzigen Aktanten für die Frage nach der Bezeichnung dieser Aktantenfunktionen von einer Priorität der Kausal-Funktion sowohl vor der Prädikativ- als auch vor der Final-Funktion auszugehen. 
2. Der grundsätzliche Unterschied zwischen der akkusativischen, der ergativischen und der aktivischen Bezeichnung von Aktantenfunktionen läßt sich auf der Grundlage einer ausschließlichen Berücksichtigung der Prädikativ- und der Kausal-Funktion darstellen, wobei sich als Darstellungsmittel die vierstellige Schemastruktur (a) anbietet. Da weder eine Schemastruktur (a):

\begin{tabular}{|l|c|c|}
\cline { 2 - 3 } \multicolumn{1}{c|}{} & Prädikativ-Funktion & Kausal-Funktion \\
\hline $\begin{array}{l}\text { in syntagmatischer } \\
\text { Isolierung }\end{array}$ & $\mathrm{P}$ & $\mathrm{C}$ \\
\hline $\begin{array}{l}\text { in syntagmatischem } \\
\text { Kontrast }\end{array}$ & $\mathrm{P}(\mathrm{C})$ & $\mathrm{C}(\mathrm{P})$ \\
\hline
\end{tabular}

nach syntagmatischer Isolierung versus syntagmatischem Kontrast unterschiedene Bezeichnung ein und derselben Aktantenfunktion noch eine unterschiedliche Bezeichnung der Prädikativ- und der Kausal-Funktion im Fall der syntagmatischen Isolierung sehr ökonomisch wären, und da andererseits eine gleichzeitige Nicht-Bezeichnung beider Oppositionen zur Nicht-Unterscheidung von $P(C)$ und $C(P)$ und damit zu gravierenden Verwechslungsgefahren führen würde, kann es nicht überraschen, daß weder Beispiele fủr einen alle vier Fälle differenzierenden noch solche für einen ïberhaupt nichts differenzierenden Bezeichnungstyp bekannt sind. Wofür Beispiele vorliegen oder zumindest vorzuliegen scheinen, sind die in den folgenden Schemata 1 bis 4 wiedergegebenen vier Bezeichnungstypen und die in ihnen gefundenen KompromiBlösungen zwischen dem Bestreben nach ökonomie und dem nach der Vermeidung von Verwechslungsgefahren.

Zu den in diesen und den weiteren Schemata eingetragenen Kasusnamen ist anzumerken, daß sie der leichteren Orientierung dieren und

- weder die Unterstellung, die Unterscheidung zwischen akkusativischem, ergativischem und aktivischem Bezeichnungstyp selte nur für den Fall der Bezeichnung von Aktantenfunktionen durch Kasusgrammeme am Nomen (cf. Heger 1982b), 
- noch die Unterstellung, Kasus als Bezeichnungen von Aktantenfunktionen und die durch sie bezeichneten Aktantenfunktionen stinden in einer eineindeutigen Abbildungsbeziehung zu einander,

implizieren. Vielmehr stehen die verwendeten Kasusnamen repräsentativ fir je einzelsprachliche 'Jormalbezeichnungen', wobei die folgenden Zuordnungen zu Grunde gelegt werden (cf. Heger 1982a: $\$$ 1.); daß selbst diese Zuordnungen nicht überall durchgehalten werden können, läßt sich am Beispiel des Finnischen illustrieren (cf.ibd. $\$ 1.3$.$) .$

- Akkusativ = 'Normalbezeichnung' der Prädikativ-Funktion,

- Ergativ = 'Normalbezeichnung' der Kausal-Funktion,

- Dativ = 'Normalbezeichnung' der Final-Funktion und

- Nominativ = 'Normalbezeichnung' mindestens der im Fall der syntagmatischen Isolierung nicht unterschiedenen Aktantenfunktionen.

2.1. Eine ternäre Opposition der Bezeichnungsmittel und somit eine nur eingeschränkt ökonomische Lösung liegt in Schema 1 vor, für das als Beispiel - ob zu Recht oder Unrecht, bleibe hier dahingestellt - das Motu zitiert zu werden pflegt.

Schema 1:

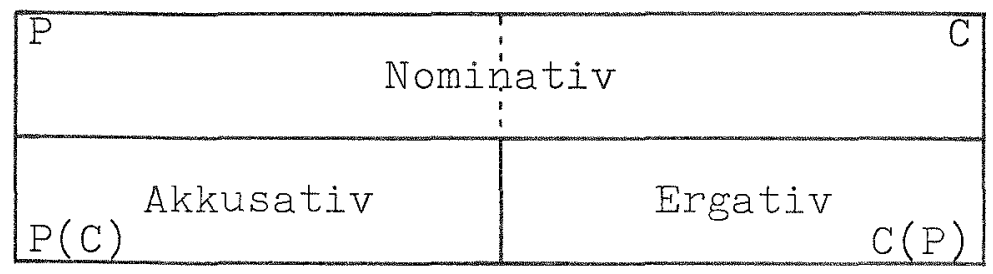

2.2. Binäre Oppositionen der Bezeichnungsmittel und somit optimale ökonomische Lösungen liegen in den folgenden drei Schemata vor, von denen Schema 2 den aktivischen, Schema 3 den akkusativischen und Schema 4 den ergativischen Bezeichnungstyp wiedergeben. Beispiele für diese drei Bezeichnungstypen sind hinlänglich bekannt und brauchen hier nicht wiederholt zu werden; worauf jedoch ausdrucklich hinzuweisen ist, ist der Umstand, daß diese Schemata nicht nur den Unterschied zwischen den genannten drei Bezeichnungstypen, 
Schema 2

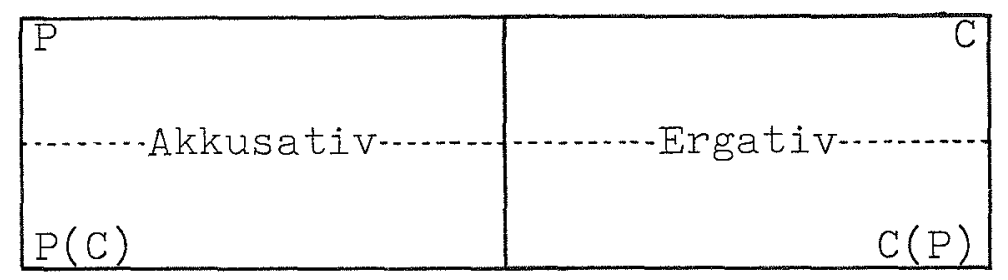

Schema 3:

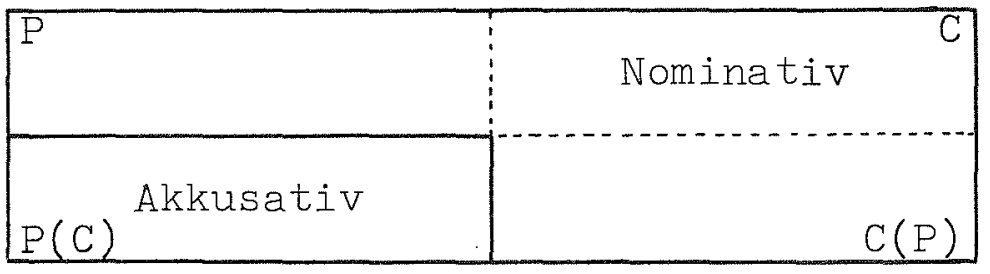

Schema 4:

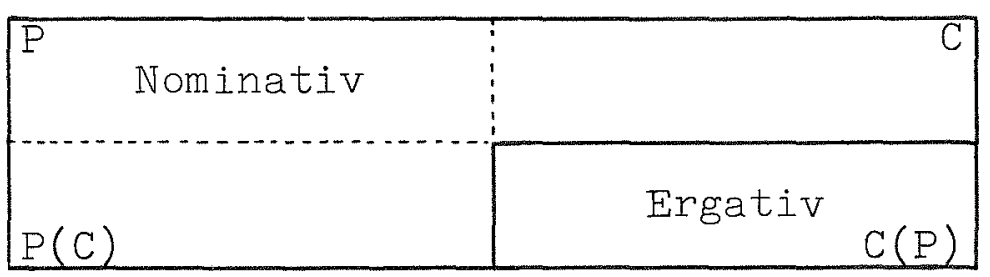

sondern auch einen Unterschied darzustellen erlauben, der zwischen zwei verschiedenen Interpretationen des Ausdrucks 'Bezeichnungstyp' zu machen ist. Bekanntlich ist in zahlreichen Sprachen eine Benutzung verschiedener Bezeichnungstypen in einer Art kombinatorischer Variation mit anderen Unterscheidungen anzutreffen, beispielsweise im Georgischen mit der Unterscheidung zwischen den Tempusgruppen des Praesens (akkusativischer Bezeichnungstyp, Schema 3) und des Aorist (ergativischer Bezeichnungstyp, Schema 4). In partieller Analogie hierzu läßt sich durchaus auch für Sprachen wie das Deutsche von dem Vorliegen einer kombinatorischen Variation mit der Diathesen-Opposition sprechen. Jedoch ist diese Analogie insofern nur partiell, als für das Georgische die Festlegung der Tempusgruppe des Aorist auf den ergativischen Bezeichnungstyp generell gilt, während für das Deutsche eine entsprechende Festlegung des Passivs auf eben diesen ergativischen Bezeichnungstyp besagt, daß zwar für $P(C)$ und $C(P)$ (= untere Schemazeile) vom Vorliegen passiver Verbalformen, fir $P$ und $C$ (= obere Schemazeile) hingegen ebenso wie im ent- 
gegengesetzten Fall des Aktivs vom Vorliegen aktiver Verbalformen auszugehen ist.

3. Ein wesentlich komplexeres Bild ergibt sich bei dè zusätzlichen Einbeziehung der Final-Funktion in die Frage nach der Unterscheidbarkeit verschiedener Bezeichnungstypen. Um die hierbei zu berücksichtigenden zwölf verschiedenen Möglichkeiten und ihre relativ üblichsten jeweiligen INormalbezeichnungen' einigermaßen übersichtlich darstellbar zu machen, werden die Darstellungen einerseits der Unterscheidung nach syntagmatischer Isolierung, syntagmatischem Kontrast mit nur einer anderen Aktantenfunktion und syntagmatischem Kontrast mit beiden anderen Aktantenfunktionen und andererseits der Unterscheidung zwischen Prädikativ-, Kausal- und Final-Funktion gemäß Schemastruktur (b) mit einander kombiniert.

Schemastruktur (b):

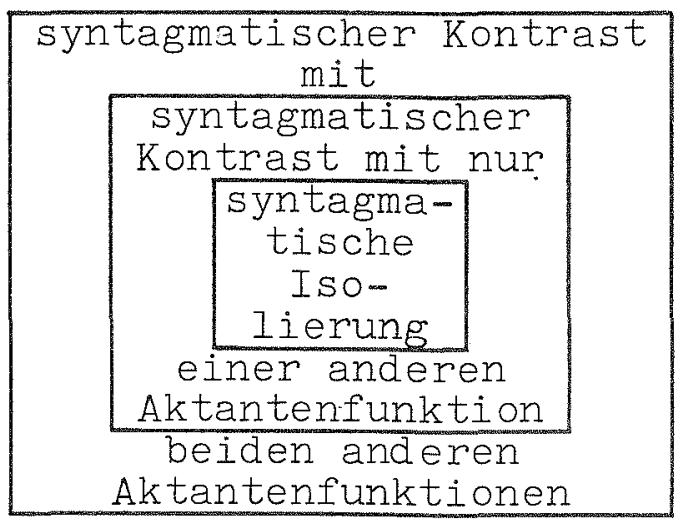
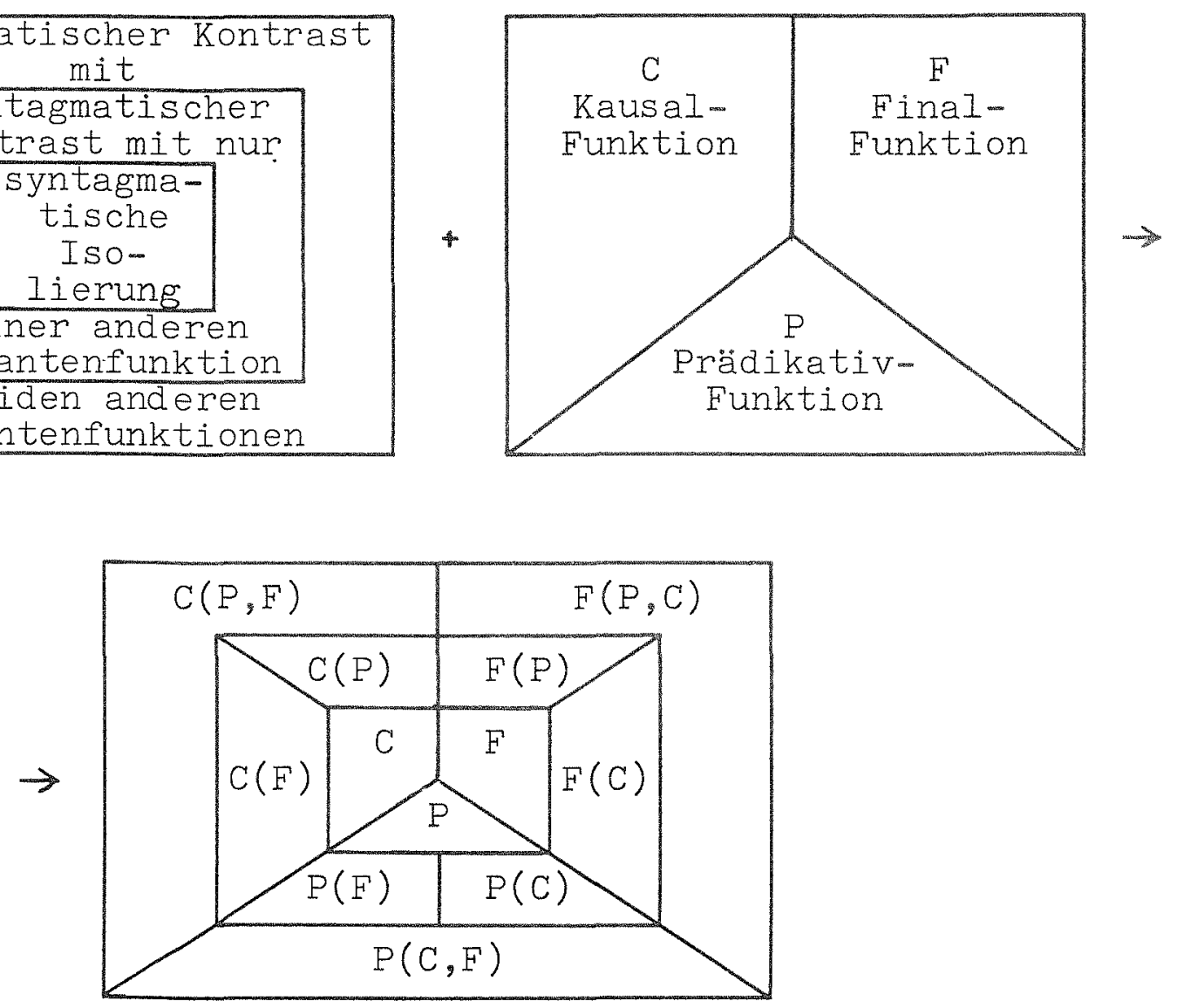
Angesichts der erheblich vergrößerten Anzahl kombinatorischer Möglichkeiten, die dieses Schema eröffnet, und angesichts der damit notwendigerweise verbundenen Relativierung des Wertes möglicher Antworten auf die Frage nach dem, was je einzelsprachlich als 'Normalbezeichnung' einzelner Schemastellen angesehen werden kann, empfiehlt es sich, vorab zu fragen, von welchen kombinatorischen Möglichkeiten im Sinne eines Kompromisses zwischen dem Streben nach ökonomie der Bezeichnungsmittel und dem nach der Vermeidung von Verwechslungsgefahren überhaupt erwartet werden kann, daß ihnen ein eigener Bezeichnungstyp entspricht. Eine Antwort auf diese Frage hat davon auszugehen, daß

- im Fall des syntagmatischen Kontrasts mit beiden anderen Aktantenfunktionen eine eigene Bezeichnung fü jede einzelne Aktantenfunktion und somit eine ternäre Opposition zur Bezeichnung der Opposition $P(C, F): C(P, F): F(P, C)$ zu erwarten sind,

- im Fall des syntagmatischen Kontrasts mit nur einer anderen Aktantenfunktion mindestens eine eigene Bezeichnung jeder der jeweils einer Verwechslungsgefahr ausgesetzten Aktantenfunktionen und somit mindestens eine binäre opposition zur Bezeichnung der Oppositionen $P(C): C(P)$, $P(F): F(P)$ und $C(F): F(C)$ (deren Glieder in Schemastruktur (b) an jeweils einander symmetrisch entgegengesetzter stelle erscheinen) zu erwarten sind, und

- im Fall der syntagmatischen Isolierung eine jeweils eigene Bezeichnung verschiedener Aktantenfunktionen nicht erforderlich ist.

Darüberhinaus ist bei der Frage nach möglichen INormalbezeichnungen' einzelner Schemastellen auch zu bericksichtigen, ob deren interne Plausibilität eine hinreichend hohe Vorkommenswahrscheinlichkeit und damit eine alle konkurrierenden Bezeichnungsmöglichkeiten eindeutig überwiegende INormalbezeichnung' uiberhaupt erwarten läßt. So wird zumindest für die Bezeichnung der Opposition $C(F): F(C)$ außerhalb von Sonderfällen wie einerseits den in 1.1. und 1.2. dargestell- 
ten und andererseits dem der Nicht-Bezeichnung eines Aktanten in Prädikativ-Funktion ein besonderes Bedürnis nicht gerade sehr häufig vorliegen und die Beantwortung der Frage nach einer 'Normalbezeichnung' dieser beiden Schemastellen daher in besonderem Maße erschwert sein. Ein Beispiel für eine noch relativ wohl etablierte 'Normalbezeichnung' dieses Falles bietet die Kasusverteilung Nominativ $\cong C(F)$ und Akkusativ $\cong F(C)$ in demjenigen Subsystem des Deutschen, das durch die Verben vom Typ beliefern, beschenken etc. gebildet wird ( $c f$. noch unten 3.2.2. und 3.2.3.).

Auf der Grundlage dieser Überlegungen lassen sich die folgenden Gruppen von erwartbaren Bezeichnungstypen bilden:

3.1. Der konsequenten Weiteranwendung des dem aktivischen Bezeichnungstyp (Schema 2) zu Grunde liegenden Prinzips der Priorität der eindeutigen Bezeichnung von Aktantenfunktionen entspricht der in Schema 5 wiedergegebene Bezeichnungstyp.

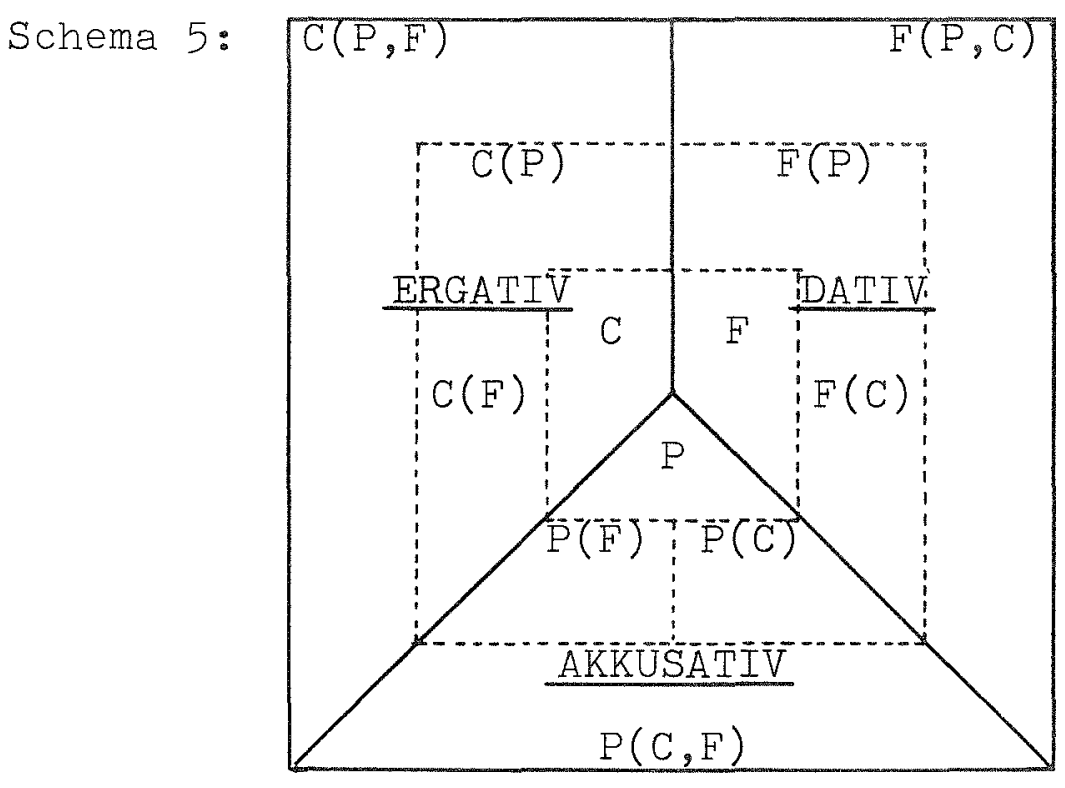

Unter den üblicherweise als Beispiele für den aktivischen Bezeichnungstyp aufgeführten Sprachen dürften Fälle, die dieser seiner konsequenten Variante entsprechen, am ehesten in den Golf-Sprachen (cf. Klimov 1974: pp.19-23) aufzufinden sein. 
3.2. Der konsequenten Weiteranwendung des sowohl dem akkusativischen als auch dem ergativischen Bezeichnungstyp zu Grunde liegenden Prinzips der Priorität der ökonomischen Ausnutzung der wegen fehlender syntagmatischer Kontraste entfallenden Bezeichnungsbedirfnisse entsprechen alle diejenigen Lösungen, in denen $P, C$ und $F$ nicht unterschieden werden, für $P(C): C(P), P(F): F(P)$ und $C(F): F(C)$ eine einzige binäre Opposition benutzt wird und lediglich fur $P(C, F): C(P, F): F(P, C)$ eine ternäre Opposition vorliegt. Da bei drei zu unterstellenden verschiedenen Bezeichnungen insgesamt sechs verschiedene hierarchische Abfolgen möglich sind, ergeben sich hierbei automatisch die in den Schemata 6 bis 9, 11 und 12 wiedergegebenen sechs homogenen Varianten. Als homogene Varianten werden dabei diejenigen Verteilungen der drei verschiedenen Bezeichnungen angesehen, in denen die jeweilige hierarchische Abfolge ausnahmslos eingehalten ist. Dieser Fall liegt vor, wenn

- die Bezeichnung 1 (die dann gemäß dem einleitend zu 2. Gesagten per definitionem ein Nominativ ist) sämtliche Vorkommen der in hierarchischer spitzenstellung stehenden Aktantenfunktion I - also I, I(II), I(III) und I(II,III) - , die beiden übrigen Aktantenfunktionen in syntagmatischer Isolierung - also II und III - und die in hierarchischer Drittstellung stehende Aktantenfunktion III zusätzlich dann, wenn sie in syntagmatischem Kontrast mit der in hierarchischer Zweitstellung stehenden Aktantenfunktion II erscheint - also III(II), und zwar in Opposition zu dem von Bezeichnung 2 bezeichneten II(II) - , bezeichnet,

- die Bezeichnung 2 die drei syntagmatisch nicht isolierten Vorkommen der in hierarchischer Zweitstellung stehenden Aktantenfunktion II - also II(I), II(III) und II(I,III) - und die in hierarchischer Drittstellung stehende Aktantenfunktion III dann, wenn sie in syntagmatischem Kontrast mit der in hierarchischer Erststellung stehenden Aktantenfunktion I erscheint - also III(I), und zwar in opposition zu dem von Bezeichnung 1 bezeichneten I(II) - , bezeichnet, und 
- die Bezeichnung 3 die in hierarchischer Drittstellung stehende Aktantenfunktion III ausschließlich dann bezeichnet, wenn sie in syntagmatischem Kontrast mit beiden anderen Aktantenfunktionen erscheint - also III (I,II).

Diesem Fall entsprechen die sechs verschiedenen hierarchi-. schen Abfolgen der Schemata 6 bis 9,11 und 12 in der folgenden Verteilung der Aktantenfunktionen P, C und F:

\begin{tabular}{|r|c|c|c|c|c|c|}
\hline Schema & 6 & 7 & 8 & 9 & 11 & 12 \\
\hline Aktantenfunktion I & P & P & C & C & F & F \\
\hline Aktantenfunktion II & C & F & P & F & P & C \\
\hline Aktantenfunktion III & F & C & F & P & C & P \\
\hline
\end{tabular}

Schemata 10 und 13 hingegen bieten zwei Beispiele für die zahlreichen heterogenen Mischformen, die aus diesen sechs homogenen Varianten geiildet werden können.

3.2.1. Bei hierarchischer Spitzenstellung der Bezeichnung der Prädikativ-Funktion ergeben sich die in cien Schemata 5 und 7 wiedergegebenen beiden Varianten des ergativischen Bezeichnungstyps. Die in Schema 6 wiedergegebene Variante

Schema 6:

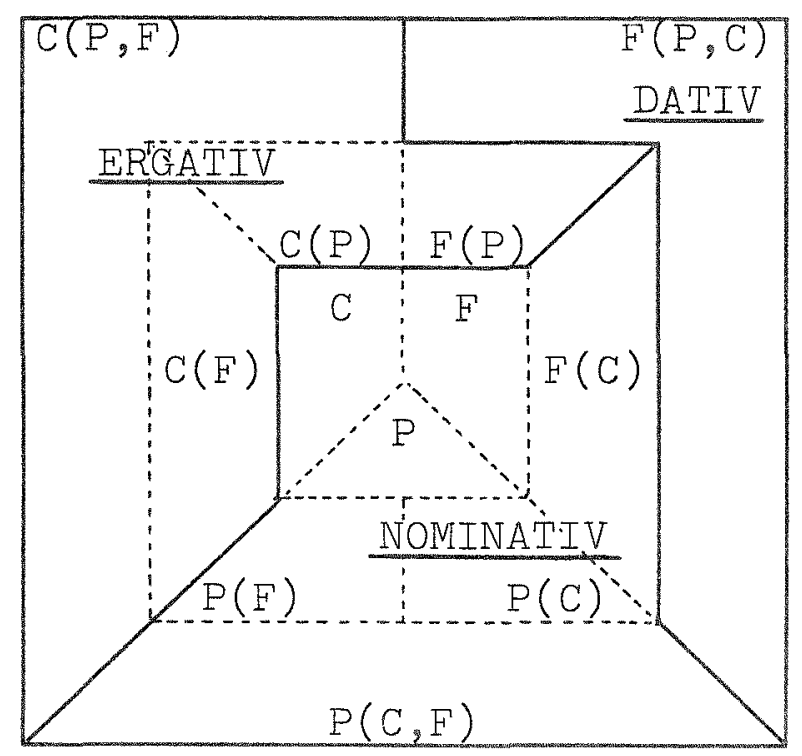

Schema 7:

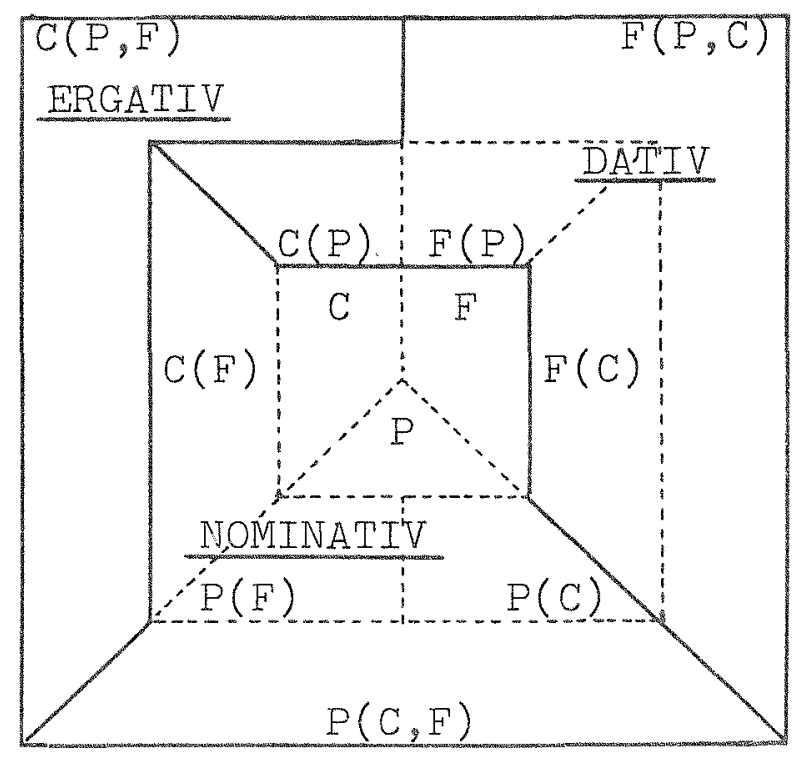


mit hierarchischer Zweitstellung der Bezeichnung der KausalFunktion liegt beispielsweise im Baskischen sowie in kombinatorischer Variation mit dem Passiv (bei Unterstellung passiver Verbalformen für alle Fälle syntagmatischen Kontrasts und aktiver Verbalformen für diejenigen syntagmatischer Isolierung) in Sprachen wie dem Deutschen vor. Fïr die in Schema 7 wiedergegebene Variante mit hierarchischer Zweitstellung der Bezeichnung der Final-Funktion sind mir keine Beispiele bekannt.

3.2.2. Bei hierarchischer Spitzenstellung der Bezeichnung der Kausal-Funktion ergeben sich die in den Schemata 8 und 9 wiedergegebenen beiden Varianten des akkusativischen Bezeichnungstyps. Die in Schema 8 wiedergegebene Variante mit

Schema 8:

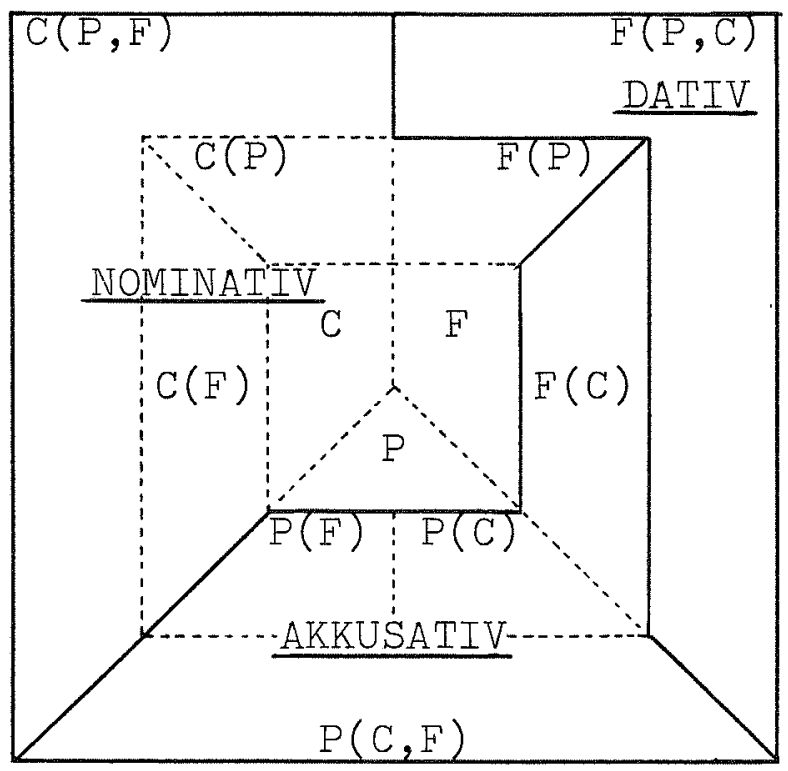

Schema 9:

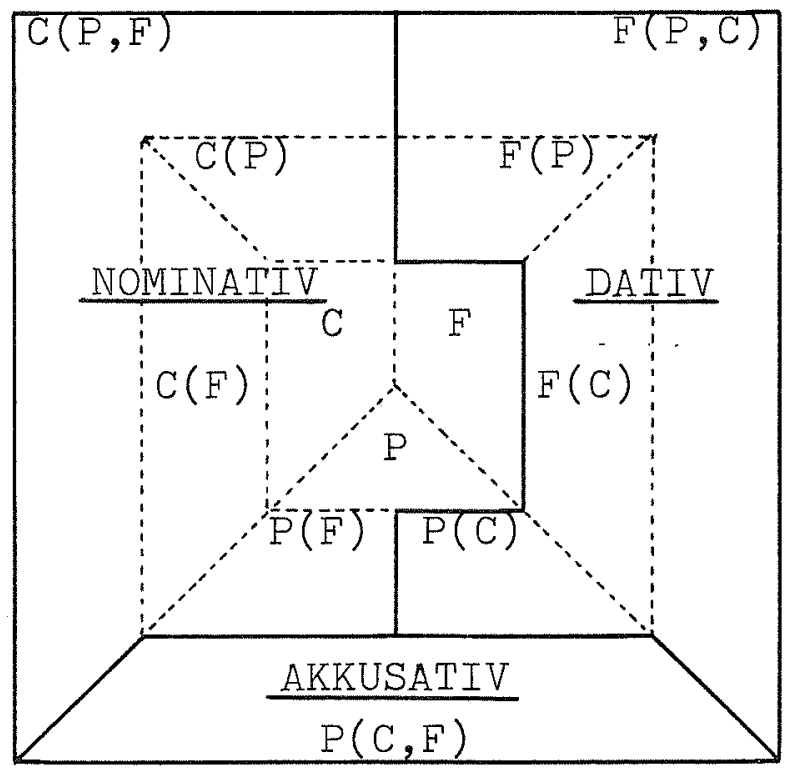

hierarchischer Zweitstellung der Bezeichnung der PrädikativFunktion entspricht genau derjenigen hierarchischen Abfolge, die der von Lucien Tesnière (cf. Tesnière 1959) vorgeschlage-

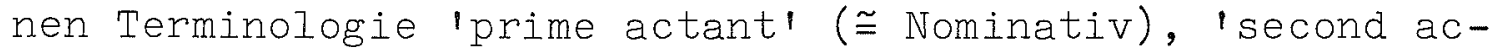

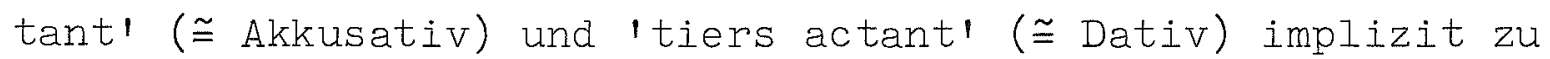
Grunde liegt. Bezogen auf die Frage nach der Herkunft dieser Hierarchie ist es gewiB kein Zufall, daß Beispiele für die ihr entsprechende Variante des akkusativischen Bezeichnungstyps in den romanischen und germanischen sprachen (in kombi- 
natorischer Variation mit dem Aktiv) den Normalfall bilden. Für die in Schema 9 wiedergegebene zweite Variante des akkusativischen Bezeichnungstyps mit hierarchischer Zweitstellung der Bezeichnung der Final-Funktion sind mir keine ihr vollständig entsprechenden Beispiele bekannt. Hingegen findet sich für die in Schema 10 wiedergegebene KompromiBlösung,

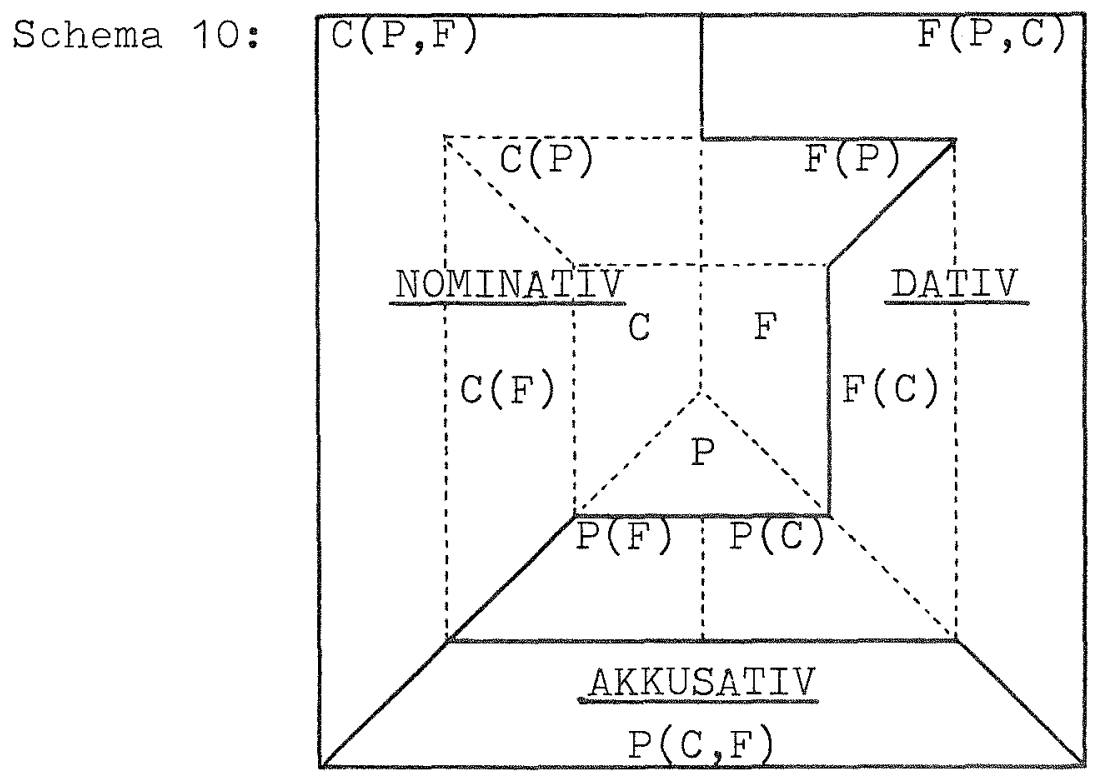

die für die Fälle des syntagmatischen Kontrasts mit nur einer anderen Aktantenfunktion der in Schema 8 wiedergegebenen, im übrigen jedoch der in Schema 9 wiedergegebenen Variante entspricht, ein Beispiel in dem oben erwähnten Subsystem, das im Deutschen die Verben vom Typ beliefern, beschenken etc. bilden - allerdings mit einer in zwei von drei Fällen von der sonst gültigen Terminologie abweichenden Kasusverteilung: nur dem NOMINATIV in Schema 10 entspricht der deutsche Nominativ, dem dortigen DATIV hingegen der deutsche Akkusativ und dem dortigen AKKUSATIV eine Art Instrumentalis (der $C$ beliefert den $F$ mit dem $P$ ).

3.2.3. Bei hierarchischer Spitzenstellung der Bezeichnung der Final-Funktion ergeben sich die in den Schemata 11 und 12 wiedergegebenen Varianten eines erst jetzt neben dem ergativischen und dem akkusativischen zusätzlich auftauchenden Bezeichnungstyps, der mangels eines besseren Terminus einstweilen 'dativisch' genannt sei. Während mir für die in diesen 
Schema 11:

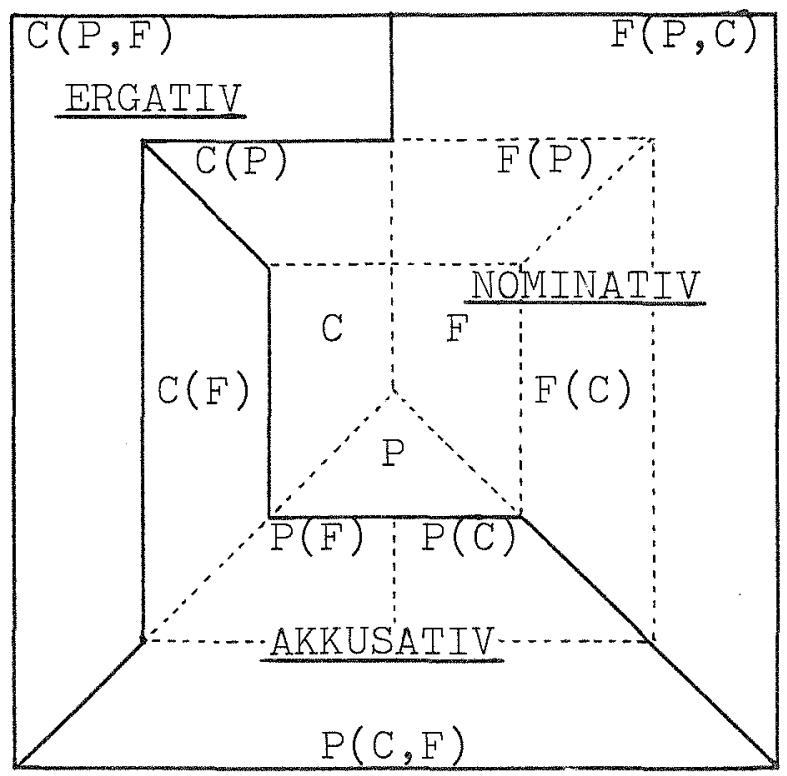

Schema 12:

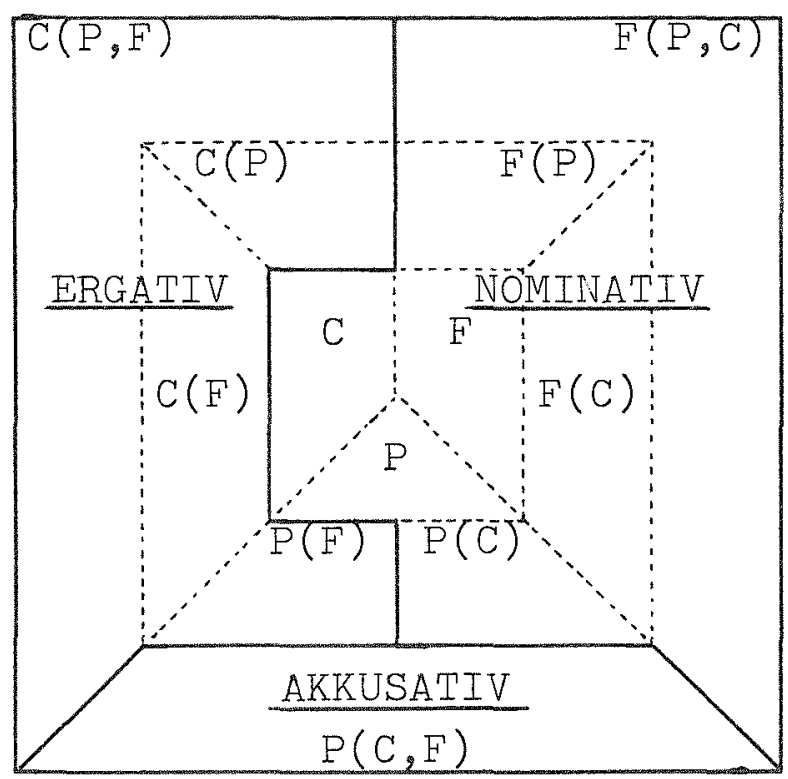

beiden Schemata wiedergegebenen Varianten selbst (mit hierarchischer Zweitstellung der Bezeichnung der Prädikativ-Funktion in Schema 11 und derjenigen der Kausal-Funktion in Schema 12) keine Beispiele bekannt sind, läßt sich auch hier wieder eine in Schema 13 wiedergegebene KompromiBlösung, die in

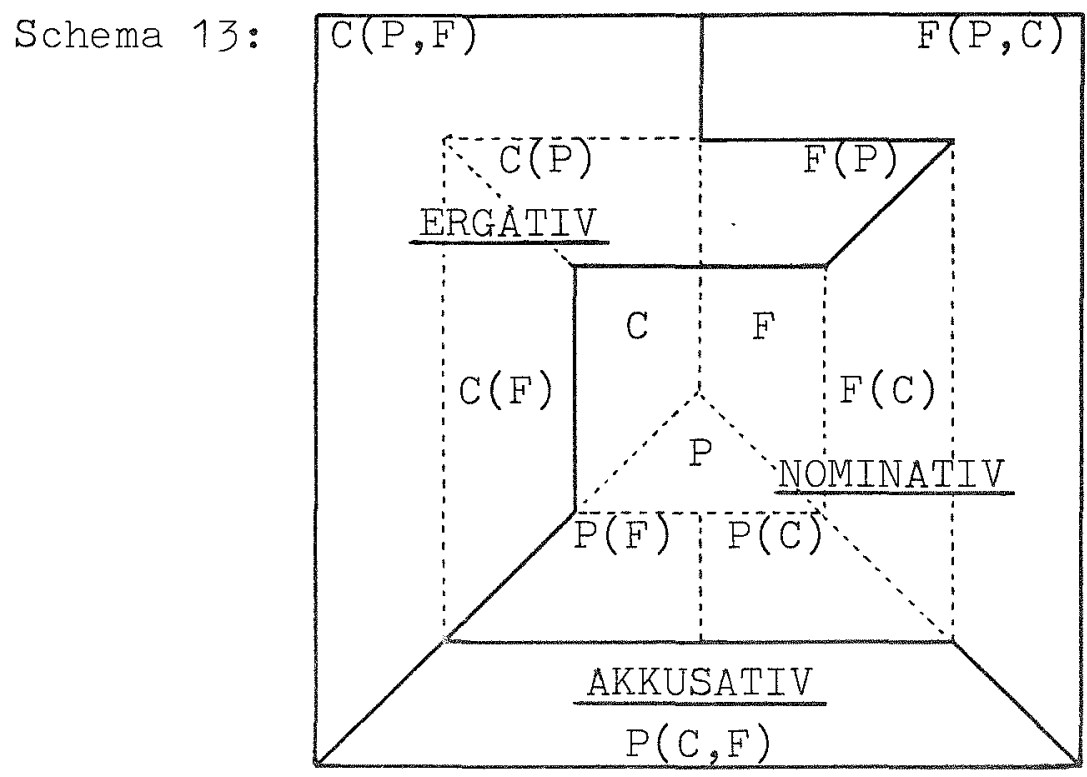

den Fällen des syntagmatischen Kontrasts mit nur einer anderen Aktantenfunktion der hierarchischen Spitzenstellung der Bezeichnung der Final-Funktion widerspricht, im übrigen jedoch mit der in schema 12 wiedergegebenen Variante mit hier- 
archischer Zweitstellung der Bezeichnung der Kausal-Funktion übereinstimmt, durch zwei Sonderfälle im Deutschen illustrieren. Es sind dies

- einerseits diejenige kombinatorische Variation mit der Diathesenopposition, bei der fưr den Fall des sylltagmatischen Kontrasts mit beiden anderen Aktantenfunktionen das l bekommen'-Passiv (der $F$ hat den $P$ von der C geliefext bekommen), fïr die Fälle des syntagmatischen Kontrasts mit nur einer anderen Aktantenfunktion das normale ('werden!-..)Passiv und fir diejenigen syntagmatischer Isolierung aktive Verbalformen zu unterstellen sind; und

- andererseits das schon für die Exemplifizierung von Schema 10 herangezogene Subsystem der Verben vom Typ beizefern, beschenken etc. in diesmal zusätzlicher kombinatorischer Variation mit dem Passiv (d.h. bei Unterstellung passiver Verbalformen für alle Fälle syntagmatischen Kontrasts und aktiver Verbalformen für diejenigen syntagmatischer Isolierung); wie schon bei Schema 10 tritt auch hier die als Instrumentalis eingestufte $\mathrm{P}(\mathrm{C}, \mathrm{F})$-Bezeichnung an der stelle des AKKUSATIVs in Schema 13 ein (der ist won dem $C$ mit dem $p$ beliefert worden).

3.3. Neben einerseits der konsequenten Weiterbilaung des aktivischen Bezeichnungstyps in Schema 5 und andererseits den sechs homogenen Varianten einer konsequenten Weiterbildung des ergativischen und des akkusativischen Bezeichnungstyps sowie den in den Schemata 10 und 13 nur angedeuteten zahlreichen von diesen eröffneten Kompromißmöglichkeiten stehen mindestens ebenso zahlreiche weitere Kombinationsmöglichkeiten, die sich als KompromiBlösungen zwischen der konaequenten Weiteranwendung der Prinzipien einerseits der Priorität der eindeutigen Bezeichnung von Aktantenfunktionen (Schema 5) und andererseits der Priorität der ökonomischen Ausnutzung der wegen fehlenden syntagmatischen Kontrasts entfallenden Be-zeichnungsbedüfnisse (Schemata 6 bis 13) beschreiben lassen. Unter ihnen scheinen am ehesten diejenigen eine Aussicht auf Exemplifizierbarkeit rilt tatsächlichen Beispielen zu eröff- 
nen, die für die Final-Funktion dem ersten und für die Prädikativ- und die Kausal-Funktion dem zweiten Prinzip folgen. Diese somit als Kombinationen aus einem aktivischen Bezeichnungstyp für die Final-Funktion und entweder einem ergativischen oder einem akkusativischen Bezeichnungstyp für die Prädikativ- und die Kausal-Funktion einzustufenden Bezeichnungstypen liegen virtuell überall dort vor, wo Verben, die auf Grund ihrer semantischen Analyse einen Aktanten in Prädikativ-Funktion und einen in Final-Funktion implizieren, - oder zumindest bestimmte Subklassen von ihnen wie die verba sentiendi oder die Possessiv-Verben (haben/gehoren) - eine andere Kasusrektion aufweisen als die 'transitiven' Verben mit Aktanten in Prädikativ- und in Kausal-Funktion. GewissermaBen als Standardformen dieser kombinierten Bezeichnungstypen ist das zu erwarten, was als Kombination von ergativischem und aktivischem Bezeichnungstyp in Schema 14 und als Kombination von akkusativischem und aktivischem Bezeichnungstyp in Schema 15 wiedergegeben ist. Beide Schemata - oder hinsicht-

Schema 14:

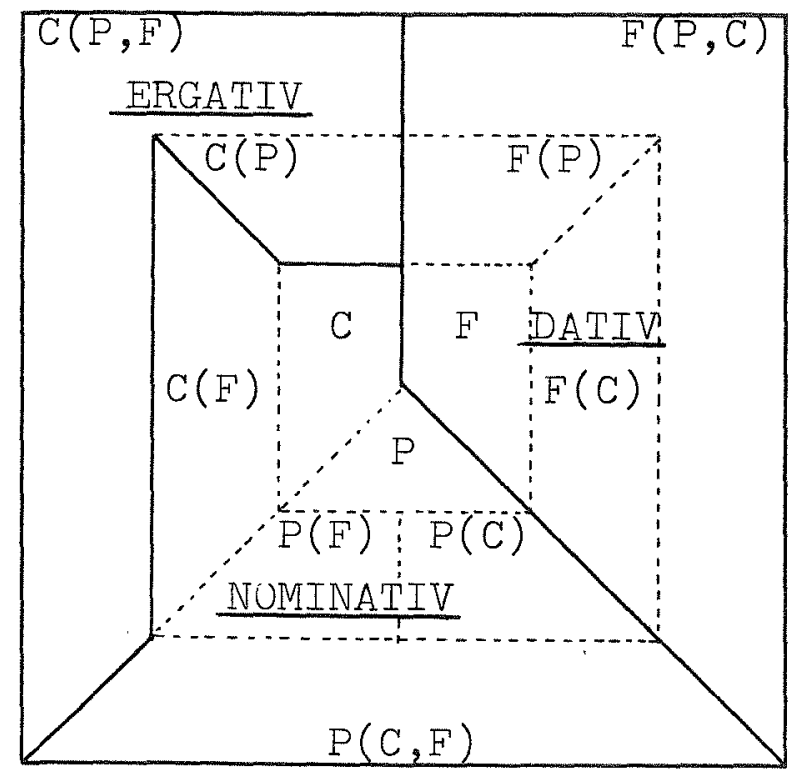

Schema 15:

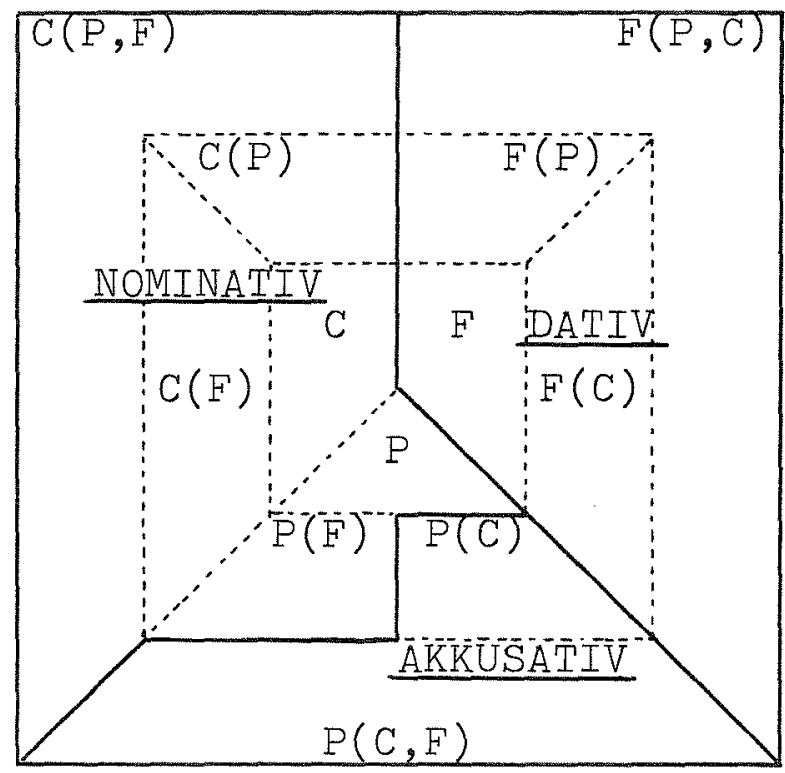

lich der Schemastellen $F, F(C)$ und $C(F)$ gebührend zu modifizierende Varianten von ihnen - dürften sich als geeignete Darstellungen der im Georgischen in kombinatorischer Variation mit der Opposition von aoristischer und praesentischer 
Tempusgruppe als Normalfall vorliegenden Bezeichnungstypen und entsprechend Schema 14 als geeignete Darstellung des beispielsweise im Avarischen als Normalfall vorliegenden Bezeichnungstyps erweisen. Schema 15 kann darüberhinaus an den von Schema 8 abweichenden Stellen durch die - allerdings kaum mehr als 'Normalfälle' einstufbaren - deutschen Kasusverteilungen in dem $F$ ist kalt, der $P(F)$ gehort dem $F(P)$ und der $C(F)$ iefert dem $F(C)$ illustriert werden.

4. Der Verzicht auf die explizite Vorfuhrung der in dieser oder anderen Kombinationen des aktivischen mit dem ergativischen, dem akkusativischen und/oder dem 'dativischen' Bezeichnungstyp möglichen Varianten erfolgt im Vertrauen darauf, daß das bislang Ausgefihrte ausreicht, um

- einerseits den Nutzen des Operierens mit als einzelsprachunabhängigen noematischen Kategorien definierten Aktantenfunktionen als tertium comparationis im Vergleich verschiedener Bezeichnungstypen sei es einer einzigen oder sei es verschiedener Einzelsprachen - beispielsweise an Hand des grundlegenden Unterschieds zwischen den oft gleichgesetzten Verhältnissen einerseits im Baskischen (Schema 6) und andererseits in kaukasischen Sprachen (Schema 14) - sichtbar zu machen,

- andererseits aber auch deutlich werden zu lassen, daß bei der schnell wachsenden Zahl theoretisch denkbarer kombinatorischer Möglichkeiten, wie sie hier an Hand einer geringen Auswahl aus dem, was schon die Berücksichtigung lediglich dreier verschiedener Aktantenfunktionen eröffnet, hinreichend sichtbar geworden sein düfte, nur mehr in sehr eingeschränktem Maße damit gerechnet werden kann, daß in einer gegebenen Einzelsprache charakteristische 'Normalbezeichnungen' anzutreffen seien.

Ein noch weitergehend differenzierendes Vorgehen düfte sich daher wesentlich eher als für eine Sprachtypologie für eine Typologie einzelner Verbalrektionen als angemessen erweisen. Als Andeutung des Ausmaßes, in welchem schon die hier im 
dritten Abschnitt gebotenen Darstellungen zwischen diesen beiden Bereichen stehen, mag die abschließende Übersicht über die im Deutschen im nur in den Beispielsätzen 2, 18b, 19 und 20b durchbrochenen Rahmen eines einzigen semantischen Bereichs anzutreffenden Bezeichnungsmöglichkeiten dienen. Aus ihr ist abzulesen, daß zwar der Ausschluß von Ergativ und Dativ als Bezeichnungen der Prädikativ-Funktion und von Akkusativ und Dativ als Bezeichnungen der Kausal-Funktion als (einzel)sprach-spezifisch angesehen werden kann, daß hingegen alle übrigen Kasusverteilungen nur mehr bestimmten Subsystemen entsprechen, die insgesamt als grammematisch und/oder lexematisch spezifizierte Diathesen eingestuft werden können:

\begin{tabular}{|c|c|c|c|c|}
\hline & Nominativ & Akkusativ & Dativ & Ergativ \\
\hline $\mathrm{P}$ & 1 & 2 & & \\
\hline$P(C)$ & 3 & 4 & & \\
\hline$P(F)$ & 5,6 & 7 & & \\
\hline$P(C, F)$ & 8 & $9,10,(11)$ & & \\
\hline$C$ & 12 & & & 13 \\
\hline$C(P)$ & 4 & & & 3 \\
\hline$C(F)$ & 14,15 & & & 16,17 \\
\hline$C(P, F)$ & 9,11 & & & 8,10 \\
\hline$F$ & 18 & 19 & 20 & \\
\hline$F(P)$ & 7 & & 6 & 5 \\
\hline$F(C)$ & 16 & 14 & 15,17 & \\
\hline$F(P, C)$ & 10 & $(11)$ & $8,9,(11)$ & \\
\hline
\end{tabular}

Die Einklammerung des Verweises auf Beispielsatz 11 betrifft die oben in 3.2.2. im Zusammenhang mit Schema 10 erwähnten Abweichungen von der normalen Kasus-Terminologie. 
Beispielsätze:

1 Der $P$ ist bekannt.

Es regnet den $\mathrm{P}(\mathrm{z} \cdot \mathrm{B}$. Hunde und Katzen).

3 Der $P$ wird von dem $C$ verkündet.

4 Den $\mathrm{P}$ verkündet der $\mathrm{C}$.

5 Der $\mathrm{P}$ wird von dem $\mathrm{F}$ gekannt.

6 Der $P$ ist dem $F$ bekannt.

7 Den $\mathrm{P}$ kennt der $\mathrm{F}$.

8 Der $\mathrm{P}$ wird von dem C dem F mitgeteilt.

9 Den $P$ teilt der $C$ dem $F$ mit.

10 Den P bekommt von dem C der F mitgeteilt.

11 Über den $P$ unterrichtet der $C$ den $F$.

12 Der C predigt.

13 Von dem $\mathrm{C}$ wird gepredigt.

14 Der C unterrichtet den $F$.

15 Der C predigt dem $F$.

16 Von dem $C$ wird der $F$ unterrichtet.

17 Von dem C wird dem F gepredigt.

$18 \mathrm{a}$ Der $\mathrm{F}$ Dekommt gepredigt. b Der F friert. 19

Den F frient.

20a Dem F wird gepredigt.

b Dem ist kalt. 
Bibliographie

Heger 1976 = Klaus Heger, Monem, Wort, Satz und Text. Tübingen 1976.

Heger 1982a = Klaus Heger, "Nominativ - Subjekt - Thema". In: Fakten und Theorien - Festschrift für Helmut Stimm zum 65.Geburtstag. Hrsg. von Sieglinde Heinz und Ulrich Wandruszka. Tübingen 1982. 87-93.

Heger 1982b = Klaus Heger, "'il la lui a donnée, à Jean, son père, sa motol - Neue Überlegungen zu einem alten Beispiel". In: Festschrift für Johannes Hubschmid zum 65. Geburtstag - Beiträge zur allgemeinen, indogermanischen und romanischen Sprachwissenschaft. Hrsg. von Otto Winkelmann und Maria Braisch. Bern 1982. 53-66.

Klimov $1974=$ Georgij A. Klimov, "On the Character of Languages of Active Typology". In: Linguistics 131, 1974, $11-25$.

Tesnière 1959 = Lucien Tesnière, Eléments de syntaxe structuraze. Paris 1959. 
In der Reihe akup erscheinen die Arbeiten des Kölner Universalienprojekts (DFG-Projekt, Leitung Prof. Dr. Hansjakob seiler). Die Nummern 1-15 sind erschienen als Linguistic Workshop I-III (LW I, LW II, LW III), München: Fink 1973-75.

1. Seiler, H. 1973, "Das Universalienkonzept", LW I, 6-19.

2. Lehmann, C. 1973, "Wortstellung in Fragesätzen", LW I, 20-53.

3. Ibañez, R. 1973, "Programmatische Skizze: Intonation und Frage", LW I, 54-61.

4. Brettschneider, G. 1973, "Sexus" im Baskischen: Die sprachliche Umsetzung einer kognitiven Kategorie", LW I, 62-72.

5. Stephany, U. 1973, "Zur Rolle der Wiederholung in der sprachlichen Kommunikation zwischen Kind und Erwachsenen", LW I, 73-98.

6. Seiler, H. 1974, "The Principle of Concomitance: Instrumental, Comitative and Collective (With special reference to German)" , LW II, 2-55.

7. Seiler, H. 1974, "The Principle of Concomitance in Uto-Aztecan", LW II, 56-68.

8. Lehmann, C. 1974, "Prinzipien für "Universal 14"" LW II, 69-97.

9. Lehmann, C. 1974, "Isomorphismus im sprachlichen Zeichen", LW II, 98-123.

10. Seiler, H. 1975, "Die Prinzipien der deskriptiven und der etikettierenden Benennung", LW III, 2-57

11. van den Boom, H. 1975, "Zum Verhältnis von Logik und Grammatik am Beispiel des neuinterpretierten $\lambda$ - operators", LW III, 58-92.

12. Untermann, J. 1975, "Etymologie und Wortgeschichte", LW III, 93-116.

13. Lehmann, C. 1975, "Strategien für Relativsätze", LW III, 117-156.

14. Ultan, R. 1975, "Infixes and their origins", LW III, 157-205.

15. Stephany, U. 1975, "Linguistic and extralinguistic factors in the interpretation of children's early utterances", LW III, 206-233. 
16. Ultan, R. 1975. "Descriptivity grading of Finnish bodypart terms".

17. Lehmann, C. 1975. "Determination, Bezugsnomen und Pronomen im Relativsatz".

18. Seiler, H. 1975. "Language Universals and Interlinguistic Variation".

19. Holenstein, E. 1975. "Semiotische Philosophie?".

20. Seiler, H. 1976. "Introductory Notes to a Gramar of Cahuilla" (To appear in Iinguistic Studies offered to Joseph Greenberg on the occasion of his 60th birthay).

21. Ultan, R. 1976, "Descriptivity in the Domain of BodyPart Terms".

22. van den Boom, H. 1976. "Bedeutungsexplikation und materiale Implikation".

23. Seiler, H. 1977(a). "The Cologne Project on Language Universals: Questions, Objectives, anä Prospects". Seiler, H. 1977 (b). "Determination: A Functional Dimension for Interlanguage Comparison" (final version of Seiler, H. 1976. "Determination..." published as akup 23, 1976.

(To appear in: Papers from the Gummersbach Conference on Language Universals. The Hague: Mouton).

24. Moshinsky, J, 1976. "Measuring Nominal Descriptivity" .

25. Seiler, H. (ed.) 1976. "Materials for the DFG International Research Conference on Language Universals".

26. Walter, H. 1976. "Das Problem der Deskriptivität am Beispiel deutscher Verbalderivation".

27. Seiler, H. 1977. "Two Systems of Cahuilla Kinship Expressions: Labeling and Descriptive" (To appear in the Festschrift for Madison $S$. Beeler).

28. Holenstein, E. 1977. "Motive der Universalienforschung".

29. Virkkunen, P. 1977. "Zum Ausdruck der notivischen Bestimmtheit im Finnischen. Mit einer schluBbemerkung zum typologischen Vergleich des Französischen und des Finnischen von Wolfgang Raible".

30. Kölver, U. 1977. "Nominalization and Lexicalization in Modern Newari".

31. van den Boom, H. 1978. "Paradigmenwechsel als Notationswechsel: Saussure - Chomsky".

32. Holenstein, E. 1978. "Von der Hintergehbarkeit der Sprache (und der Erlanger Schule)". 
33. Ramat, Paolo. 1978. "Y a-t-il une typologie profonde? (quelques considérations théoriques (et pratiques)".

34. Kölver, Ulrike. 1979. "Syntaktische Untersuchung von Numeralklassifikatoren im Zentralthai".

35. Holenstein, Elmar. 1979. "Zur Begrifflichkeit der Universalienfoschung in Linguistik und Anthropologie".

36. Lehmann, Christian. 1979. "Der Relativsatz. Typologie seiner Strukturen. Theorie seiner Funktionen. Kompendium seiner Grammatik".

37. Serzisko, Fritz. 1980. "Sprachen mit Zahlklassifikatoren: Analyse und Vergleich".

38. Barron, Roger. 1980. "Das Phänomen klassifikatorischer Verben in nordamerikanischer Indianersprachen: Ein typologischer Versuch".

39. Seiler, H. 1980. "Two Types of Cahuilla Kinship Expressions: Inherent and Establishing".

40. Stachowiak, Franz-Josef. 1981. "Zum funktional-operationalen Ansatz in der sprachlichen Universalienforschung aus psycholinguistischer sicht".

Lehmann, Christian. 1981. "On some current views of the language universal".

Serzisko, Fritz. 1981. "Gender, noun class and numeral classification: a scale of classificatory techniques".

41. Clasen, Berndt. 1981. "Inhärenz und Etablierung".

42. Seiler, Hansjakob. 1981. "POSSESSION as an Operational Dimension of Language".

43. Seiler, Hansjakob. 1982. "Possessivity, Subject and Objekt." 44. Mosel, Ulrike. 1982. "Possessive Constructions in Tolai".

45. Lehmann, Christian. 1982. "Rektion und syntaktische Relationen".

46. Lehmann, Christian. 1982. "Twenty-four questions on Iincuistic typology and a collection of answers".

47. Heine, Bernd/ Reh, Mechthild. 1982. "Patterns of grammaticalization in African languages".

48. Lehmann, Christian. 1982. "Thoughts on Grammaticalization. A programmatic sketch. Vol. I".

49. Kölver, Ulrike. 1983. "Indonesische Verbalpräfixe. Ein Beitrag zur Dimension INHÄRENZ und ETABLIERUNG".

50. Mosel, Ulrike. 1983. "Adnominal and Predicative Possessive Constructions in Melanesian languages".

51. Ostrowski, Manfred. 1983. "Zur Nomen:Verb-Rolationierung im wogulischen, Jurakischen und Jukagirischen". 
52. van den Bơom, Holger. 1983. "Zum Verhältnis von Logik und Linguistik im Bezug auf UNITYP-Grunasätze".

53. UNITYP-Forschergruppe. 1983. "Beiträge zum Problembereich Skalen und Kontinua". 